\title{
Sporadic inclusion body myositis—diagnosis, pathogenesis and therapeutic strategies
}

\author{
Marinos C Dalakas
}

\section{SUMMARY}

Sporadic inclusion body myositis (sIBM) presents with a characteristic clinical phenotype of slow-onset weakness and atrophy, affecting proximal and distal limb muscles and facial and pharyngeal muscles. Histologically, sIBM is characterized by chronic myopathic features, lymphocytic infiltrates invading non-vacuolated fibers, vacuolar degeneration, and accumulation of amyloid-related proteins. The cause of sIBM is unclear, but two processes - one autoimmune and the other degenerative-appear to occur in parallel. In contrast to dystrophies, in SIBM the autoinvasive $\mathrm{CD}^{+} \mathrm{T}$ cells are cytotoxic and antigen-driven, invading muscle fibers expressing major histocompatibility complex class I antigen and costimulatory molecules. The concurrent degenerative features include vacuolization, filamentous inclusions and intracellular accumulations of amyloid- $\beta$-related molecules. Although viruses have not been amplified from the muscle fibers, at least 12 cases of sIBM have been seen in association with retroviral infections, indicating that a chronic persistent viral infection might be a potential triggering factor. Emerging data imply that continuous upregulation of cytokines and major histocompatibility complex class I on the muscle fibers causes an endoplasmic reticulum stress response, resulting in intracellular accumulation of misfolded glycoproteins and activation of the transcription factor NFKB, leading to further cytokine activation. In spite of the brisk, antigen-driven T-cell infiltrates, sIBM does not respond to immunotherapies. New therapies using monoclonal antibodies against lymphocyte signaling pathways might prove helpful in arresting disease progression.

KEYWORDS autoimmune, degeneration, immunotherapies, inflammatory myopathies, sporadic inclusion body myositis

\section{REVIEW CRITERIA}

I searched for peer-reviewed original articles in PubMed published from 1995 to 2005. The search terms used were "polymyositis", "inclusion body myositis" and "inflammatory myopathies”. All papers identified were English-language, full-text papers.

\section{Dalakas is Chief of the Neuromuscular Diseases Section, National} Institute of Neurological Disorders and Stroke, National Institutes of Health, Bethesda, MD, and Professor of Clinical Neurology at George Washington University, Washington DC, USA.

\section{Correspondence}

Neuromuscular Diseases Section, National Institute of Neurological Disorders and Stroke, National Institutes of Health, Bethesda, MD 20892-1382, USA

dalakasm@ninds.nih.gov

\section{Received 20 December 2005 Accepted 30 May 2006}

www.nature.com/clinicalpractice

doi:10.1038/ncpneuro0261

\section{INTRODUCTION}

Sporadic inclusion body myositis (sIBM) is one of the three main subsets of inflammatory myopathies, the other two being polymyositis and dermatomyositis. ${ }^{1-5}$ All of these conditions are characterized by inflammation in the endomysium (hence the term inflammatory myopathies), muscle fiber necrosis, elevation of serum muscle enzymes and a varying degree of muscle weakness. Dermatomyositis can occur in children and adults, has a subacute onset, and affects muscles and skin. Polymyositis is also a subacute myopathy that affects adults but rarely affects children, and presents with proximal muscle weakness. By contrast, sIBM is a slowly progressive myopathy that affects proximal and distal muscles, and has a distinctive histopathology characterized by autoimmune inflammatory features combined with degenerative features, such as vacuoles, filamentous inclusions (hence the term inclusion body myositis) and accumulation of amyloid-related proteins.

sIBM is often misdiagnosed as polymyositis or other diseases, and is frequently only suspected retrospectively when a patient with presumed polymyositis does not respond to therapies. ${ }^{1}$ This became evident 15 years ago when we began recruiting patients who had been diagnosed with therapy-resistant polymyositis to a controlled study that investigated treatment with highdose intravenous immunoglobulin. This study was never completed, because it transpired that most of the patients actually had sIBM.

This review outlines our current knowledge of sIBM, including clinical presentation, diagnosis, pathogenesis and therapeutic strategies.

\section{CLINICAL FEATURES OF SPORADIC INCLUSION BODY MYOSITIS}

sIBM is the most common acquired myopathy in patients above the age of 50 years, and it affects men slightly more often than it does women. Its prevalence is estimated at between 4.3 and 9.3 per $1,000,000$, rising to 35.3 per $1,000,000$ for people over the age of 50 years. 6,7 
sIBM causes weakness and atrophy of the distal and proximal muscles, and involvement of quadriceps and deep finger flexors are clues to early diagnosis. ${ }^{2}$ Patients often present with falls because their knees collapse owing to quadriceps muscle weakness, or with difficulty performing certain tasks, such as turning keys, tying knots and holding golf clubs, owing to weakness of finger flexors. Neck flexors and extensors are frequently affected; head drop and camptocormia are rare manifestations. Dysphagia occurs in up to $60 \%$ of patients with sIBM, leading to choking episodes. Facial muscle weakness is common and at times prominent, although the extraocular muscles are not affected. Sensory function is usually normal; mildly diminished vibratory sensation at the ankles is sometimes observed, but this is presumed to be age-related, or attributable to a comorbidity. The tendon reflexes, although preserved early in the disease, can diminish in the late stages as the atrophy of major muscle groups becomes evident. The distal weakness does not represent a neurogenic process, but rather a primary myopathy that affects proximal and distal muscles, as confirmed by macroelectromyography. ${ }^{8}$ The weakness and atrophy are sometimes asymmetric, resembling a lower motor neuron disease. Disease progression is slow but steady, and resembles that of a dystrophy. Most patients with sIBM require an assistive device, such as a cane, walker or wheelchair, within several years of onset. ${ }^{9}$

\section{DIAGNOSIS OF SPORADIC INCLUSION BODY MYOSITIS}

The clinical diagnosis of sIBM is confirmed by muscle biopsy, and is aided by electromyography (EMG) and determination of serum muscle enzyme levels. Creatine kinase levels can initially be elevated up to 10 -fold, but they remain only slightly elevated as the disease progresses. EMG shows increased spontaneous activity, with fibrillations, complex repetitive discharges and positive sharp waves. The voluntary motor units are myopathic, with low-amplitude polyphasic units, usually of short (but, in a few rare cases, long) duration. MRI of the forearms and quadriceps might show the selective patterns of muscle involvement even before the manifestation of clinical signs, ${ }^{10}$ but it is of limited diagnostic value.

Muscle biopsy is essential for diagnosis: from the outset, there are signs of chronicity characterized by hypertrophic, atrophic and split fibers with internal nuclei and increased connective tissue, indicating that the disease process has begun long before the patient seeks medical attention. The main histological features are multifocal lymphocytic infiltrates invading non-necrotic muscle fibers ${ }^{1-5}$ vacuoles in fibers not invaded by lymphocytes (these 'rimmed vacuoles' contain basophilic granular deposits, consisting of membranous whorls, around the edges), and Congo-red-positive amyloid deposits. The amyloid deposits are best visualized with Texas red fluorescent filters, but can also be seen quite clearly with crystal violet (Figure 1A,B). ${ }^{1-5,11}$ Abnormal mitochondria characterized by ragged red fibers or cytochrome-oxidase-negative fibers are frequent (Figure 1C). Electron microscopy reveals accumulation of $15-21 \mathrm{~nm}$ tubulofilamentous inclusions and cytoplasmic collections of 6-10 nm amyloid-like filaments that immunoreact with various amyloid-proteinrelated antibodies. ${ }^{5}$ Abnormal myonuclei with intranuclear $7 \mathrm{~nm}$-wide filaments are also detected in up to $3.5 \%$ of the nuclei, but their significance in vacuolar formation remains unclear. The vacuoles are not unique to sIBM, and are also seen in other myopathies, such as dysferlinopathies, myofibrillar myopathies, facioscapulohumeral muscular dystrophy and Emery-Dreifus muscular dystrophy, and even in chronic neurogenic conditions such as old poliomyelitis. ${ }^{12,13}$ The vacuoles are most easily identified on frozen sections stained with hematoxylin and eosin or trichrome stain; paraffin processing dissolves the granular material and the vacuoles are often missed. In some specimens, the inflammation or the vacuoles are sparse, necessitating a second biopsy from a different site.

If a patient has the typical clinical phenotype of sIBM, but the muscle biopsy shows only features of a chronic inflammatory myopathy (inflammation, large fibers, splitting, and increased connective tissue, but no vacuoles), the diagnosis is probable sIBM. ${ }^{3,14}$ If, however, there is also strong upregulation of major histocompatibility complex (MHC) class I antigens (see below), and amyloid deposits and cytochrome-oxidase-negative fibers are present (Figure 1), the diagnosis of sIBM is rather more certain.

\section{Differential diagnosis}

Shaking hands with a patient can provide the first indication of sIBM, because of the weak 

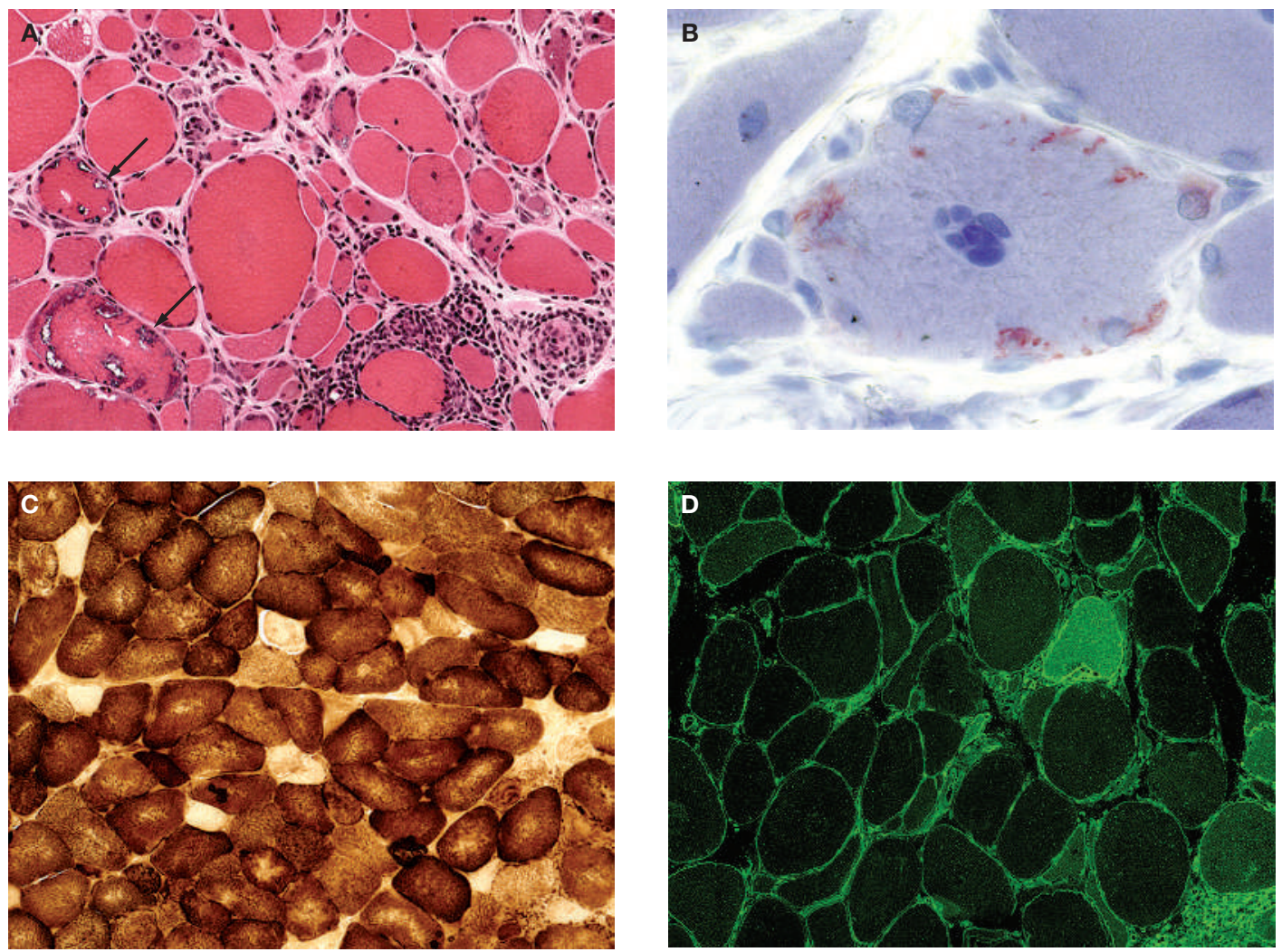

Figure 1 Characteristic histological features in cross-sections of muscle biopsies in patients with sporadic inclusion body myositis. (A) Inflammation and vacuoles. Note endomysial inflammation, with lymphocytes invading non-necrotic, healthy-appearing muscle fibers, and 'red-rimmed' vacuoles in two muscle fibers (arrows) not invaded by inflammatory cells. If the course of the same vacuolated fibers is followed at considerable length in longitudinal sections, they remain devoid of autoinvasive inflammatory T cells. (B) Intracellular deposits of amyloid, easily identified with crystal violet stain on frozen sections.

(C) Scattered cytochrome-oxidase-negative fibers, indicative of abnormal mitochondrial function.

(D) Strong major histocompatibility complex class I expression (green) in all fibers, regardless of whether they are invaded by T cells.

grip. If the patient complains of falls due to weakness at the knees and feet, has atrophic thighs, and does not report paresthesias or cramps, sIBM is very likely. Diagnostic dilemmas arise when the weakness and atrophy are slightly asymmetric or limited to the lower extremities, raising the possibility of a lower motor neuron disease. Motor neuron disorders, however, can be distinguished from IBM by the presence of hyperreflexia, cramps, fasciculations and large motor units on EMG.

sIBM is most often misdiagnosed as polymyositis, based on the simplistic view that creatine kinase elevation with endomysial inflammation denotes polymyositis. In contrast to sIBM, however, polymyositis starts subacutely (over weeks to months, compared with months to years in sIBM), causes proximal and symmetrical muscle weakness, and is often associated with higher creatine kinase levels. In addition, the biopsy lacks the chronicity signs of sIBM. The distinction between sIBM and the other, mostly hereditary vacuolar myopathies, such as myofibrillar myopathies or hereditary inclusion body myopathy with mutations in the glucosamine (UDP-N-acetyl)-2-epimerase/ $\mathrm{N}$-acetylmannosamine kinase (GNE) gene, might be more subtle because these myopathies also affect distal muscles and the vacuoles are identical to those of sIBM. Endomysial inflammation accompanied by MHC class I expression, and the aforementioned typical clinical phenotype in patients above the age of 50 years, should, however, point to sIBM. 
Box 1 Factors supporting an immunopathogenic disease mechanism for sporadic inclusion body myositis.

1 Immunogenetic association with $D R \beta 1^{*} 0301$, $D Q \beta 1{ }^{*} 0201$ alleles and the B8-DR3-DR52-DQ2 haplotype

2 DQ2 haplotype; the human leukocyte antigen (HLA)-A haplotype is associated with earlier disease onset ${ }^{15-17}$

3 Occurrence of sporadic inclusion body myositis (sIBM) in family members of the same generation (familial inflammatory IBM), as seen with other autoimmune disorders ${ }^{20}$

4 Association with other autoimmune disorders and autoantibodies ${ }^{17,23}$

5 Association with paraproteinemia at a significantly higher frequency than in age-matched controls $(22.8 \% \text { vs } 2 \%)^{25}$

6 Association with common variable immunodeficiency and natural killer cells ${ }^{24}$

7 Association with HIV and human T-lymphotropic virus (HTLV)-1 infection (13 cases reported to date) ${ }^{26,46-48}$

$8 \mathrm{CD}^{+}$autoinvasive T cells surround major histocompatibility complex (MHC) class I-expressing fibers, express perforin and activation markers of cytotoxicity, and are clonally expanded ${ }^{31-33,37-39}$

9 Ubiquitous upregulation of $\mathrm{MHC}$ class I antigen and costimulatory molecules on muscle fibers, even those not invaded by T cells; the counter-receptors of the costimulatory molecules are overexpressed on the autoinvasive $T$ cells ${ }^{28,40,41}$

10 Strong upregulation of cytokines, chemokines and their receptors at the protein, messenger RNA and gene level ${ }^{42-45}$

\section{DISEASE MECHANISMS IN SPORADIC INCLUSION BODY MYOSITIS}

sIBM is a complex disorder, the underlying cause of which is unclear. The observation that the intact muscle fibers are invaded by $\mathrm{T}$ lymphocytes, whereas the vacuolated fibers lack T-cell autoinvasion but show strong expression of MHC class I antigens, has led to the suggestion that two processes might occur in parallel: a primary immune process due to T-cell-mediated cytotoxicity, and a non-immune process characterized by vacuolization and intracellular accumulation of amyloid-related molecules, probably due to MHC class I-induced stress. An interplay between inflammatory mediators and stressor or degenerative molecules is likely.

\section{Immunogenetics}

Genetic factors are presumed to play a role in sIBM, on the basis of an association between sIBM and certain human leukocyte antigen (HLA) genes, especially the $D R \beta 1^{*} 0301$ and $D Q \beta 1^{*} 0201$ alleles. ${ }^{15}$ Alleles of the 8.1 ancestral haplotype in the center of the MHC class II region seem to confer susceptibility to IBM. ${ }^{16}$ The B8-DR3-DR52-DQ2 haplotype is found in $67 \%$ of sIBM patients, similar to the frequency of this haplotype in myasthenia gravis. ${ }^{17}$ The B8-DR3-DR52-DQ2 haplotype is also associated with earlier disease onset, indicating that immunoregulatory genes are inherently connected with the manifestation of symptoms. Apolipoprotein E $\varepsilon 4$ has also been associated with sIBM, ${ }^{18}$ but no linkage to amyloid precursor protein or prion protein genes has been observed. ${ }^{19}$

The features of sIBM have been observed in siblings in several families, a condition that has been termed familial inflammatory sIBM. ${ }^{20}$ These cases are distinct from the hereditary inclusion body myopathies (hIBM), a heterogeneous group of non-inflammatory, inherited syndromes. ${ }^{21}$ A subset of hIBM that spares the quadriceps muscles results from mutations in the GNE gene. ${ }^{22}$

\section{Autoimmune features}

Evidence for an autoimmune basis for sIBM is supported by numerous observations (Box 1), including association with other autoimmune diseases, ${ }^{17,23}$ common variable immunodeficiency, ${ }^{24}$ and autoantibodies in similar frequencies to those seen in classic autoimmune disorders. ${ }^{17,23}$ Also, as in many other autoimmune disorders, sIBM occurs in family members of the same generation, ${ }^{20}$ and there is a strong immunogenetic association, as described above. ${ }^{15-17}$ sIBM is associated with paraproteinemias in up to $22.8 \%$ of patients-much greater than the $2 \%$ frequency found in age-matched controls-indicating disturbed immunoregulation. ${ }^{25}$ There is also an association with retroviruses (see below), ${ }^{26}$ and evidence of formation of immunological synapses between autoinvasive cytotoxic $\mathrm{T}$ cells and muscle fibers (Figure 2). The autoinvasive $\mathrm{T}$ cells are driven by specific, but still unidentified, antigens. ${ }^{27}$ 


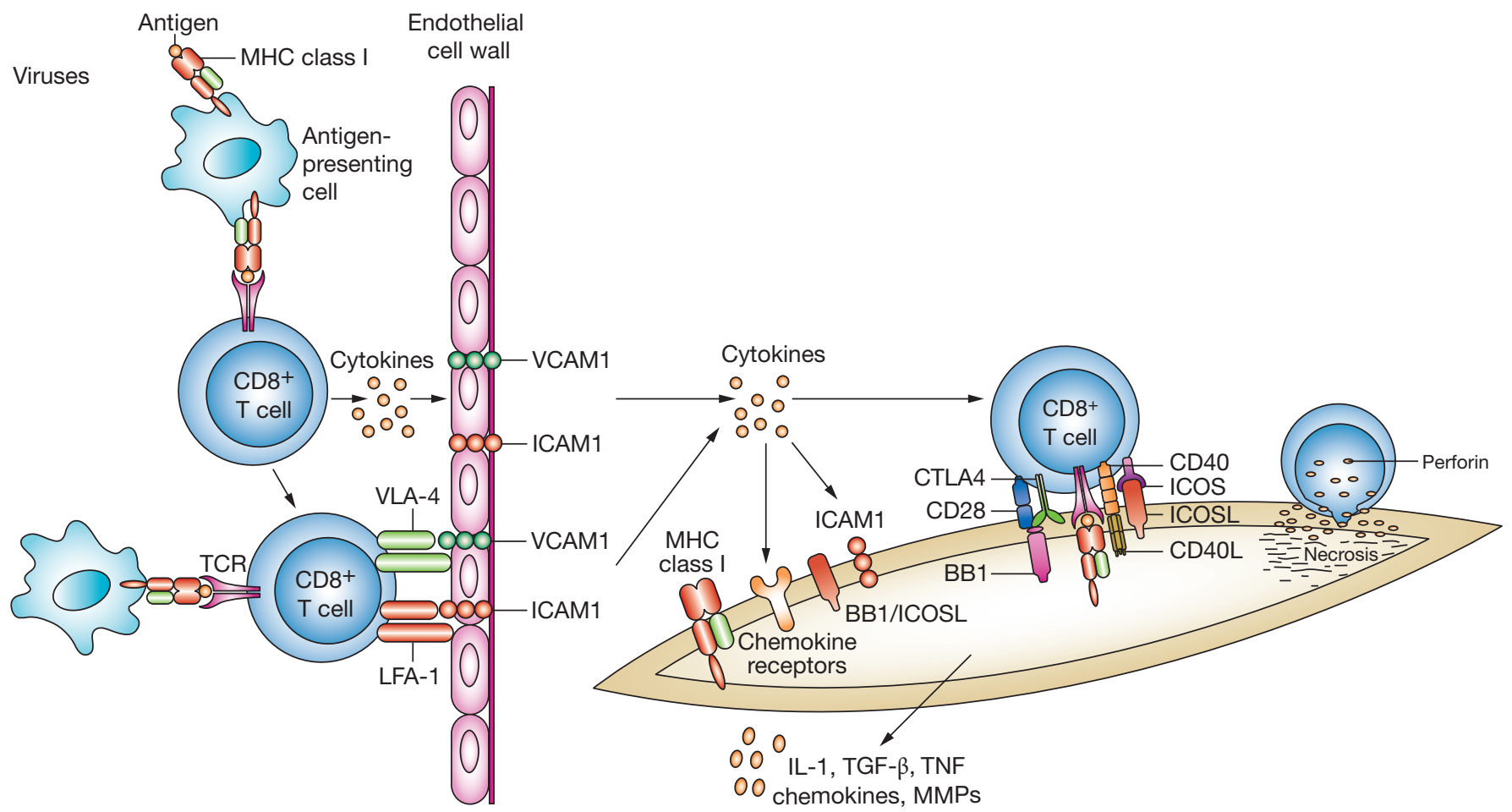

Figure 2 Sequence of immunopathological changes in sporadic inclusion body myositis. It is hypothesized that the disease begins when viruses break tolerance, and $\mathrm{CD}^{+} \mathrm{T}$ cells, on receiving putative antigens by macrophages that serve as antigen-presenting cells, become activated. The released cytokines upregulate VCAM1 and ICAM1, thereby allowing the transgression of T cells through the endothelial cell wall to reach the muscle fiber. The released cytokines upregulate MHC class I, chemokine receptors, costimulatory molecules and ICAM1 on the muscle fibers, allowing the invading T cells to scan for antigens and initiate the formation of immunological synapses between MHC class I and the T-cell receptors. The engagement of the costimulatory molecules BB1, ICOS and CD40 on the muscle fibers with their ligands CD28, CTLA4, ICOSL and CD40L on the autoinvasive T cells completes the synapse and activates the T cells. Muscle fiber necrosis occurs via perforin granules released by the autoinvasive cytotoxic T cells. The muscle fibers are also active modulators because they secrete cytokines and chemokines, which further activate the invading $\mathrm{CD}^{+} \mathrm{T}$ cells thereby contributing to the persistence and chronicity of the immunopathologic process. Abbreviations: CTLA4, cytotoxic T-lymphocyte-associated protein 4; ICAM1, intercellular adhesion molecule 1; ICOS, inducible T-cell costimulator; ICOSL, inducible T-cell costimulator ligand; IL-1, interleukin-1; LFA-1, leukocyte function-associated antigen 1; MHC, major histocompatibility complex; MMP, matrix metalloproteinase; TCR, T-cell receptor; TGF- $\beta$, transforming growth factor- $\beta$; TNF, tumor necrosis factor; VCAM1, vascular cell adhesion molecule 1; VLA-4, very late activation antigen 4.

Muscle fibers do not normally express MHC class I antigens. In sIBM, however, MHC class I is ubiquitously expressed even on muscle fibers remote from the inflammation (Figure 1D). ${ }^{4,28}$ In other chronic myopathies or dystrophies, MHC class I is either absent or only present in degenerating/regenerating fibers. Furthermore, in dystrophies, the few autoinvasive $\mathrm{T}$ cells are clonally diverse and do not express cytotoxicity markers. It is probable that in sIBM, cytokines are the main inducers of MHC class I expression, because in human myotubes, proinflammatory cytokines upregulate MHC class I. ${ }^{29}$ As we discuss later in the review, the chronic upregulation of MHC class I exerts a stressor effect in the endoplasmic reticulum (ER), which might lead to a self-sustaining T-cell response. ${ }^{30}$

The autoinvasive $\mathrm{CD} 8^{+}$cells contain perforin granules, which, on release, are known to induce cell necrosis. ${ }^{31-33}$ In sIBM, the perforin pathway, as opposed to the Fas-Fas-L-dependent apoptotic process, is the cytotoxic effector mechanism, in spite of the fact that Fas antigen is expressed on muscle fibers, and Fas- $\mathrm{L}$ is expressed on T cells. ${ }^{34,35}$

$\mathrm{T}$ cells recognize an antigen via the complementarity-determining region 3 (CDR3) of the T-cell receptors (TCRs). ${ }^{36}$ The TCRs form heterodimers of $\alpha$ and $\beta$ chains encoded by multiple gene families. During T-cell development, 
billions of different TCRs are generated by rearrangement of TCR genes in the precursor $\mathrm{T}$ cells. The progeny of a single precursor cell is called a T-cell clone, and all members of the clone express the same antigen-specific TCR with unique nucleotide sequences in the CDR3 region. ${ }^{33}$ When exposed to the relevant antigen, a clone is stimulated to divide, leading to clonal expansion. In sIBM, but not in dermatomyositis or dystrophies, only $\mathrm{T}$ cells expressing certain TCR families are recruited to the muscle from the circulation. ${ }^{37,38}$ Furthermore, the autoinvasive-but not the perivascular- $\mathrm{T}$ cells are clonally expanded, indicating that they are driven by specific antigens to invade the muscle fibers. Interestingly, such clonally expanded autoinvasive $\mathrm{T}$ cells persist over time, as confirmed with sequential muscle biopsies, even from different muscles. ${ }^{38,39}$

The MHC class I molecules present antigenic peptides to TCRs of $\mathrm{CD}^{+}$cells only in the presence of costimulatory molecules and their counter-receptors. In sIBM, the costimulatory molecules BB1 (also known as CD80 or B7-1) and ICOSL are expressed on MHC class I-positive muscle fibers, and they make cell-to-cell contact with ligands on $\mathrm{CD}^{+}$cells. $^{31,33,40,41} \mathrm{BB} 1$ and ICOSL are induced by interferon- $\gamma$ or tumor necrosis factor (TNF) on human myoblasts, ${ }^{33,41}$ and might confer antigen-presenting-cell function on the muscle fibers.

\section{T-cell transmigration, cytokine signaling and chemokine receptors}

Certain cytokines (interleukin-1 [IL-1], IL-2, IL-5, IL-10, TNF and transforming growth factor- $\beta$ $[$ TGF- $\beta]$ ) and chemokines (MCP-1, MIP- $1 \alpha$, Mig and IP-10) are variably overexpressed in sIBM, and might contribute to costimulation, T-cell activation and transmigration. ${ }^{42-44}$ The chemokine receptors CCR1-CCR5, CCR2A and CCR2B are upregulated on the endothelium and inflammatory cells, enhancing the local immune circuitry. Various adhesion molecules and their ligands are also overexpressed on the endothelium or activated T cells, and these molecules facilitate the transmigration of lymphocytes towards the muscle fibers. ${ }^{42-45}$ Because human myotubes can be stimulated by cytokines to express or secrete immunologically important soluble molecules such as TGF- $\beta$, MCP-1, MIP- $1 \alpha$, IL-1 $\beta, \beta$-amyloid precursor protein ( $\beta$-APP), cell adhesion molecules, chemokine receptors, MHC and costimulatory molecules, the muscle cells are active modulators rather than passive targets, and they contribute to the persistence or chronicity of the immunopathologic process. ${ }^{33}$

\section{Viruses as possible triggering factors}

Although paramyxoviruses and mumps have been indirectly associated with sIBM, molecular techniques have failed to confirm their presence in muscle. The best evidence points towards a connection with retroviruses. ${ }^{26}$ At least seven HIV or human T-lymphotropic virus (HTLV)-1-positive patients with sIBM have been reported, ${ }^{46-48}$ and we have seen six more cases in the past 3 years, ${ }^{26}$ indicating that the disease might be more common in patients who live longer and harbor this virus for several years. In these patients, the virus does not persist or replicate within the muscle fibers, but is present only in occasional endomysial macrophages. ${ }^{46}$ The disease is triggered by clonally driven subpopulations of activated $\mathrm{CD} 8^{+}$cells that expand in situ and invade muscle fibers expressing MHC class $\mathrm{I}$, as seen in retrovirus-negative polymyositis and sIBM. ${ }^{26,46}$ These cells are retrovirus-specific, because their CDR3 region contains aminoacid residues that are specific for viral peptide bound to HLA molecules. ${ }^{47,48}$ The retroviral infection, combined with immune recognition of the retrovirus, is sufficient to trigger the inflammatory process.

\section{INFLAMMATION AND DEGENERATION IN INCLUSION BODY MYOSITIS}

Evidence for a degenerative process in sIBM is provided by the presence of vacuoles (almost always in fibers not invaded by $\mathrm{T}$ cells) and the intracellular deposition of Congo-red-positive amyloid and $\beta$-amyloid-related molecules (e.g. $\beta$-APP, phosphorylated tau, presenilin-1, apolipoprotein $\mathrm{E}, \gamma$-tubulin, clusterin, $\alpha$-synuclein, gelsolin), and oxidative stress proteins. These accumulations are not unique to sIBM, and they are observed to a similar extent in other vacuolar myopathies. ${ }^{12,49}$ In contrast to the other vacuolar myopathies, however, the vacuolated fibers in sIBM strongly express MHC class I molecules, which have been implicated in inducing ER stress. ${ }^{50}$

The assembly and folding of MHC class I occurs in the ER. It begins with the association of a heavy chain glycoprotein with $\beta 2$ microglobulin, forming an unstable heterodimer complex that matures only when it binds to an antigenic peptide (Figure 3). ${ }^{51,52}$ Antigenic 
peptides are synthesized in the cytosol by immunoproteasomes, and are transported to the ER by the transporter associated with antigen processing (TAP). The ER maintains quality control by processing, folding and exporting MHC molecules loaded with antigen. If an MHC molecule does not bind to an appropriate antigen, the heavy chain glycoprotein is misfolded and removed from the ER and transported to the cytosol for degradation.

In sIBM, the muscle fibers are overloaded with MHC molecules, and the antigenic peptides might not undergo proper conformational change to bind to the MHC class I complex, leading to ER stress and further protein misfolding. This model is supported by several observations, including enhanced immunoproteasome activity, ${ }^{50,53}$ enhanced MHC class I assembly activity, ${ }^{54}$ misfolding of glycoproteins and a cell stress response, ${ }^{5,50}$ upstream upregulation of $\mathrm{aB}$-crystallin in intact fibers, ${ }^{55}$ and colocalization of $\alpha \mathrm{B}$-crystallin with MHC molecules (J Schmidt and MC Dalakas, unpublished observations). Such stressor effects are also seen in transgenic mice that overexpress MHC class I, indicating that overexpression of MHC class I alone might be sufficient to induce ER stress. ${ }^{50}$

Accordingly, the following mechanism for the pathogenesis of sIBM can be proposed. Triggering factors, possibly viruses, could initiate the process leading to clonal expansion of T cells and T-cell-mediated, MHC class I-restricted cytotoxicity via the perforin pathway (Figure 4). The released cytokines upregulate MHC class I molecules, which cannot exit the ER, resulting in increased levels of the 'MHC-peptide-loading complex', and an ER-stress response. The ER stress results in accumulation of misfolded amyloid-related glycoproteins and activation of the transcription factor NFkB, both of which promote expression of inflammatory-mediator genes for cytokines and chemokines, thereby further stimulating the MHC/CD8 complex and inducing a self-sustaining inflammatory response. Such crosstalk between inflammatory and degenerative processes is supported by recent observations that in sIBM muscles there is a linear relationship between the messenger RNA (mRNA) level of cytokines and chemokines and that of $\beta$-APP, Tau and ubiquitin. 36,56 Furthermore, in human myotubes, cytokines enhance the expression of $\beta$-APP, tau and ubiquitin mRNAs. ${ }^{56}$ Additionally, in the muscles of individuals with sIBM, cytokines such as IL-1 $\beta$,

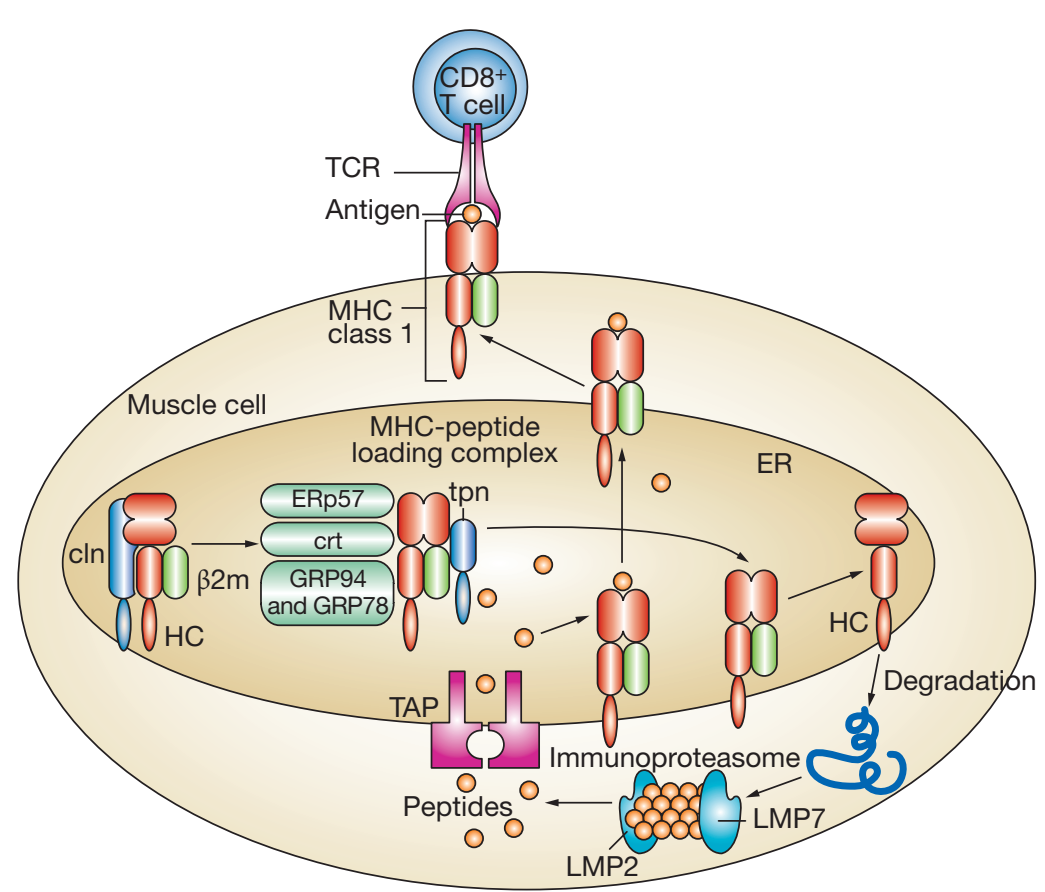

Figure 3 Assembly of MHC class I peptide complexes in the ER of the muscle fiber for antigen presentation, and translocation of misfolded $\mathrm{MHC}$ class I heavy chain glycoproteins to the cytoplasm. The heavy chain of $\mathrm{MHC}$ class I molecules associates with the chaperone protein cln, binds $\beta 2 \mathrm{~m}$, and incorporates into a complex containing chaperone proteins such as ERp57, GRP78, GRP94, crt, TAP and tPN, forming the 'MHC class I-peptide loading complex'. Antigenic peptides (derived from degradation of proteins in the cytosol and synthesized by immunoproteasomes that contain two MHC-encoded subunits, LMP2 and LMP7) are transported to the ER by TAP and bind to the MHC class I-peptide loading complex. High-affinity antigenic peptides induce a conformational change in the MHC class I molecules, thereby promoting the release of these molecules from the loading complex. ${ }^{51}$ In the muscle, the assembled MHC class I-peptide complexes traffic to the cell surface of the muscle fiber, where they are recognized by CD8 ${ }^{+} \mathrm{T}$ cells. If suitable antigenic peptides are absent, or cannot undergo the proper conformational change to bind to the MHC class I complex, the MHC class I-peptide loading complex becomes unstable, and the MHC heavy chains are misfolded and removed from the ER to the cytosol for degradation. Abbreviations: $\beta 2 \mathrm{~m}, \beta 2$ microglobulin; cln, calnexin; crt, calreticulin; $\mathrm{ER}$, endoplasmic reticulum; ERp57, glucose-regulated protein, $58 \mathrm{kDa}$; GRP78, glucose-regulated protein, $78 \mathrm{kDa}$; GRP94, glucose-regulated protein, $94 \mathrm{kDa}$; HC, heavy chain; LMP, large multifunctional protease;

$\mathrm{MHC}$, major histocompatibility complex; TAP, transporter associated with antigen processing; tpn, tapasin. Modified with permission from reference 63 (c) 2006 Nature Publishing Group.

TGF- $\beta$ and metalloproteinases co-localize with MHC class I and $\beta$-amyloid (J Schmidt and MC Dalakas, unpublished observations). Recent observations that autophagic vacuoles and protein aggregates accumulating in perinuclear inclusions, called aggresomes, are sites of viral replication, and that certain viruses induce the formation of aggresomes, ${ }^{57}$ provide credence to 


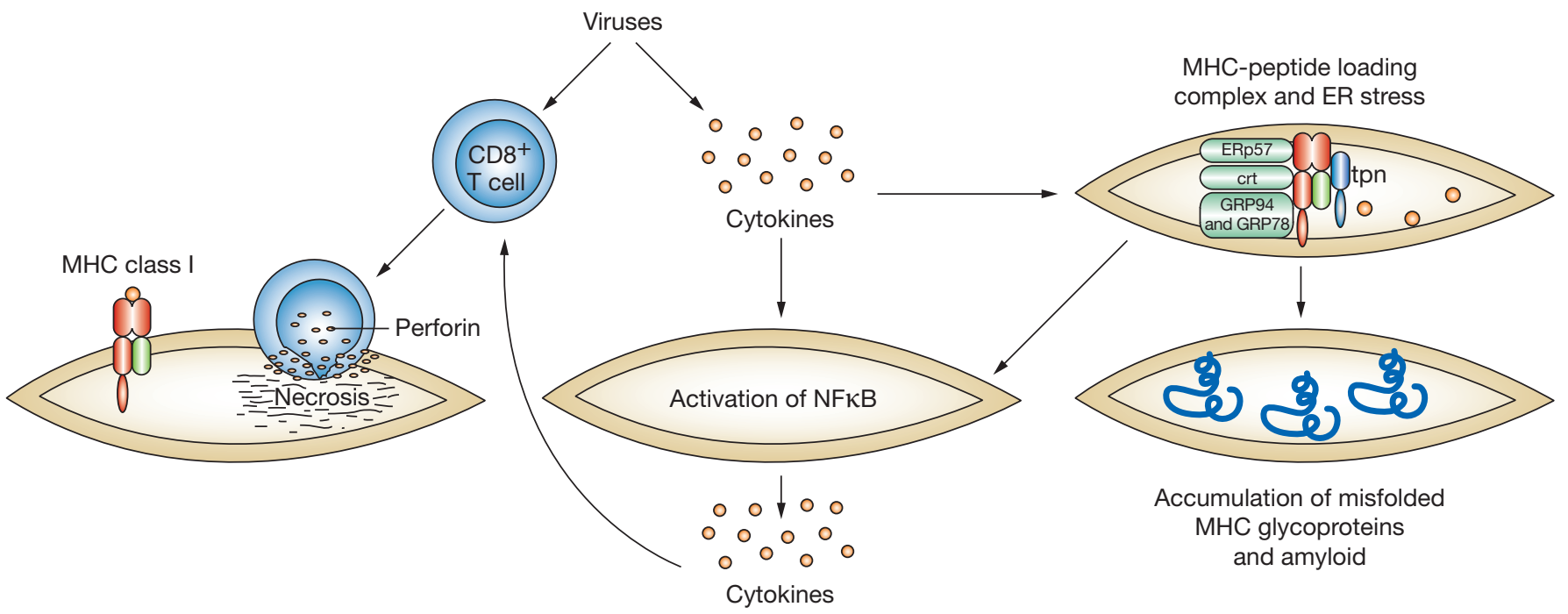

Figure 4 Proposed mechanism for the pathogenesis of inclusion body myositis. Viral triggers lead to clonal expansion of CD8 ${ }^{+} \mathrm{T}$ cells and T-cell-mediated cytotoxicity via the perforin pathway. The released cytokines upregulate MHC class I molecules and increase levels of the MHC-peptide loading complex, because the abundance of generated peptides cannot be conformationally assembled with the $\mathrm{MHC}$ to exit the ER. As a result, there is an ER stress response, which leads to activation of the transcription factor $\mathrm{NF} K \mathrm{~B}$ and accumulation of misfolded MHC glycoproteins, including amyloid-related proteins. Both NFKB and amyloid-related misfolded proteins promote the expression of inflammatory mediator genes for cytokines, the products of which further stimulate the MHC-CD8 complex, resulting in a self-sustaining inflammatory response, thereby closing the loop between inflammation and degeneration. Abbreviations: crt, calreticulin; ER, endoplasmic reticulum; ERp57, glucose-regulated protein, 58 kDa; GRP78, glucose-regulated protein, $78 \mathrm{kDa}$; GRP94, glucose-regulated protein, $94 \mathrm{kDa}$; MHC, major histocompatibility complex; tpn, tapasin.

the view that viruses could be potential triggers of sIBM, a condition in which aggresomes and autophagic vacuoles are prominent. ${ }^{58}$

The genes that encode $\beta$-APP, tau, apolipoprotein $\mathrm{E}$ and oxidative stress proteins are also overexpressed in sIBM but, as with their respective proteins, these genes are equally expressed in polymyositis, hIBM and other myopathies, indicating that the accumulated proteins represent post-translational events, probably sequelae to the chronic cell stress response. ${ }^{59,60}$ By contrast, the inflammatory mediator genes, such as those that encode adhesion molecules, cytokines, MHC, chemokines and immunoglobulin, are overexpressed in sIBM but not in hereditary IBM or the other vacuolar myopathies. ${ }^{59,60}$ In sIBM, the expression of inflammation-associated genes is even more prominent than in dermatomyositis or polymyositis. ${ }^{59,60}$

\section{THERAPEUTIC STRATEGIES}

Despite the involvement of primary immune factors in the pathogenesis of sIBM, this disease remains resistant to most immunotherapies. In this respect, sIBM resembles primary progressive multiple sclerosis, in which immune and degenerative features coexist but the disease is resistant to all therapies. At present, sIBM remains a disabling disease, with most patients requiring an assistive mobility device within 5 to 10 years of onset. In general, the older the age of onset, the more rapidly progressive is the course.

Although the common immunotherapeutic agents, such as corticosteroids, azathioprine, methotrexate, cyclosporine, cyclophosphamide and total lymphoid irradiation, are generally ineffective, some patients have responded to these therapies to a certain degree or for short periods. For example, the outcome of treatment with prednisone, together with azathioprine or methotrexate, has been largely disappointing, but some newly diagnosed patients have reported that they felt subjectively or even objectively stronger. Some patients respond to steroids, mycophenolate mofetil or intravenous immunoglobulin, and when these drugs are discontinued, the patients feel weaker. ${ }^{61}$ In three double-blind studies using intravenous immunoglobulin, up to $30 \%$ of sIBM patients experienced minimal but short-lived benefits. ${ }^{61}$ The response of dysphagia to intravenous immunoglobulin can be significant. ${ }^{61}$ 
New biologic agents targeting the main immunopathological processes depicted in Figure 2, such as T-cell proliferation, transmigration, antigen recognition or ER stress, might produce more rewarding results. Such therapies might be accomplished using the available monoclonal antibodies directed against T-cell regulatory pathways, such as CD56, costimulatory molecules (CD28/CTLA4), adhesion molecules (integrins/LFA-1/ICAM), cytokines (TNF- $\alpha$ ), chemokines or B cells (CD20). ${ }^{60}$ To this end, the results of a 12 -month, open, randomized trial in 11 sIBM patients using anti-T-lymphocyte globulin (ATG), have been encouraging, because increased strength was noted in the ATG group. ${ }^{62} \mathrm{~A}$ study using alemtuzumab, a T-cell-depleting monoclonal antibody against CD52, is currently in progress at the National Institutes of Health (MC Dalakas, Principal Investigator). Alemtuzumab is an even stronger agent than ATG, because it causes lymphocyte depletion for at least 6 months. Other promising agents include sirolimus (rapamycin), which acts via a calcineurinindependent pathway to prevent the translation of mRNA for key cytokines, and natalizumab, which blocks the transmigration of $\mathrm{T}$ cells across the endothelial cell wall. ${ }^{60}$ Agents that interfere with ER stress might protect the myofiber from chronic deleterious stimuli.

\section{CONCLUSIONS}

sIBM remains a challenging muscle disease in which inflammatory features coexist with degeneration. Although evidence indicates that there is interplay between the inflammatory mediators associated with T-cell cytotoxicity and the degeneration-associated molecules, it is unclear what triggers the $\mathrm{T}$ cells to invade muscle fibers, how the vacuoles are formed, and what role the intracellularly accumulated amyloid-related proteins have. Most remarkably, it remains a mystery why sIBM does not respond to immunotherapies in spite of the primary role of cytotoxic $\mathrm{T}$ cells in forming immunological synapses with the muscle fibers, and the strong upregulation of cytokines, chemokines and costimulatory molecules. Further studies are needed to identify the antigens that trigger T-cell activation, the pathogenic role of intracellularly accumulated proteins, and the molecular events that lead to cell stress, misfolding of glycoproteins and vacuolization. At the clinical level, the natural history of the disease needs to be established with quantitative measurements of muscle strength to assess whether newly applied therapeutic interventions can arrest disease progression. A large longitudinal study that we conducted at the NIH is now near completion. Future therapies should target T-cell signaling, costimulatory or T-cell transmigration molecules, and the factors that are responsible for the ER stress and accumulation of amyloid-related proteins.

\section{KEY POINTS}

- Sporadic inclusion body myositis (sIBM) is a slowly progressive myopathy that affects proximal and distal muscles; its histopathology is characterized by autoimmune inflammatory features combined with degenerative features

- Involvement of quadriceps and deep finger flexors are clues to early diagnosis; neck flexors and extensors are also frequently affected

- The clinical diagnosis of SIBM is confirmed by muscle biopsy, and is aided by electromyography and determination of serum muscle enzyme levels

- The cause of sIBM is unclear, but two processes-one autoimmune and the other degenerative-appear to occur in parallel

- Several cases of sIBM have been seen in association with retroviral infections, indicating that a chronic persistent viral infection might be a potential triggering factor

- Despite the involvement of primary immune factors in the pathogenesis of SIBM, this disease remains resistant to most immunotherapies; a study using alemtuzumab, a T-cell-depleting monoclonal antibody, is currently in progress

\section{References}

1 Dalakas MC (1991) Polymyositis, dermatomyositis and inclusion-body myositis. N Engl J Med 325: 1487-1498

2 Sekul EA and Dalakas MC (1993) Inclusion body myositis: new concepts. Semin Neurol 13: 256-263

3 Griggs RC et al. (1995) Inclusion body myositis and myopathies. Ann Neurol 38: 705-713

4 Mikol $\mathrm{J}$ and Engel AG (2004) Inclusion body myositis. In Myology, edn 2, 1367-1388 (Eds Engel AG and Franzini-Armstrong C) New York: McGraw-Hill

5 Askanas V and Engel WK (2003) Proposed pathogenetic cascade of inclusion-body myositis: importance of amyloid-beta, misfolded proteins, predisposing genes, and aging. Curr Opin Rheumatol 15: 737-744

6 Phillips BA et al. (2000) Prevalence of sporadic inclusion body myositis in Western Australia. Muscle Nerve 23: 970-972

7 Badrising UA et al. (2000) Epidemiology of inclusion body myositis in the Netherlands: a nationwide study. Neurology 55: 1385-1387 
8 Luciano CA and Dalakas MC (1997) Inclusion body myositis: no evidence for a neurogenic component. Neurology 48: 29-33

9 Peng A et al. (2000) Disease progression in sporadic inclusion body myositis: observations in 78 patients. Neurology 55: 296-298

10 Sekul EA et al. (1997) Magnetic resonance imaging of the forearm as a diagnostic aid in patients with sporadic inclusion body myositis. Neurology 48: 863-866

11 Mendell JR et al. (1991) Amyloid filaments in inclusion body myositis: novel findings provide insight into nature of filaments. Arch Neurol 48: 1229-1234

12 Selcen D et al. (2004) Myofibrillar myopathy: clinical, morphological and genetic studies in 63 patients. Brain 127: 439-451

13 Semino-Mora C and Dalakas MC (1988) Rimmed vacuoles with $\beta$-amyloid and ubiquitinated filamentous deposits in the muscles of patients with long-standing denervation (post-poliomyelitis muscular atrophy): similarities with inclusion body myositis. Human Pathol 29: 1128-1133

14 Amato AA et al. (1996) Inclusion body myositis: clinical and pathological boundaries. Ann Neurol 40: 581-586

15 Koffman BM et al. (1998) HLA allele distribution distinguishes sporadic inclusion body myositis from hereditary inclusion body myopathies.

J Neuroimmuno/ 84: 139-142

16 Price $\mathrm{P}$ et al. (2004) Two major histocompatibility complex haplotypes influence susceptibility to sporadic inclusion body myositis: critical evaluation of an association with HLA-DR3. Tissue Antigens 64: 575-580

17 Badrising UA et al. (2004) Associations with autoimmune disorders and HLA class I and II antigens in inclusion body myositis. Neurology 63: 2396-2398

18 Garlepp MJ et al. (1995) Apolipoprotein E epsilon 4 in inclusion body myositis. Ann Neuro/ 38: 957-959

19 Orth M et al. (2000) Sporadic inclusion body myositis not linked to prion protein codon 129 methionine homozygosity. Neurology 55: 1235

20 Sivakumar Ket al. (1997) An inflammatory, familial, inclusion body myositis with autoimmune features and a phenotype identical to sporadic inclusion body myositis: studies in three families. Brain 120: 653-661

21 Sivakumar K and Dalakas MC (1996) The spectrum of familial inclusion body myopathies in 13 families and a description of a quadriceps-sparing phenotype in nonIranian Jews. Neurology 47: 977-984

22 Eisenberg let al. (2001) The UDP-N-acetylglucosamine 2-epimerase/N-acetylmannosamine kinase gene is mutated in recessive hereditary inclusion body myopathy. Nat Genet 29: 83-87

23 Koffman BM et al. (1998) Autoimmune diseases and autoantibodies associated with sporadic inclusion body myositis. Muscle Nerve 21: 115-117

24 Dalakas MC and IIla I (1995) Common variable immunodeficiency and inclusion body myositis: a distinct myopathy mediated by natural killer cells. Ann Neuro/ 37: 806-810

25 Dalakas MC et al. (1997) Inclusion body myositis and paraproteinemia: incidence and immunopathologic correlations. Ann Neurol 41: 100-104

26 Dalakas MC (2006) Inflammatory, immune and viral aspects of inclusion-body myositis. Neurology 66 (Suppl): S33-S38

27 Dalakas MC (2002) Understanding the immunopathogenesis of inclusion-body myositis: present and future prospects. Rev Neurol (Paris) 158: 948-958

28 Karpati G et al. (1988) Expression of immunoreactive major histocapability complex products in human skeletal muscles. Ann Neurol 23: 64-72
29 Michaelis D et al. (1993) Constitutive and cytokineinduced expression of human leukocyte antigens and cell adhesion molecules by human myotubes. Am J Pathol 143: 1142-1149

30 Nagaraju K et al. (2000) Conditional up-regulation of $\mathrm{MHC}$ class I in skeletal muscle leads to selfsustaining autoimmune myositis and myositis-specific autoantibodies. Proc Natl Acad Sci USA 97: 9209-9214

31 Schmidt J et al. (2004) Upregulated inducible costimulator (ICOS) and ICOS-ligand in inclusion body myositis muscle: significance for $\mathrm{CD}^{+} \mathrm{T}$ cell cytotoxicity. Brain 127: 1182-1190

32 Goebels N et al. (1996) Differential expression of perforin in muscle-infiltrating $\mathrm{T}$ cell in polymyositis and dermatomyositis. J Clin Invest 97: 2905

33 Wiendl $\mathrm{H}$ et al. (2005) Immunobiology of muscle: advances in understanding an immunological microenvironment. Trends Immunol 26: 373-380

34 Behrens L et al. (1997) Cytotoxic mechanisms in inflammatory myopathies: co-expression of Fas and protective $\mathrm{Bcl}-2$ in muscle fibres and inflammatory cells. Brain 120: 929

35 Schneider C et al. (1996) MHC class I mediated cytotoxicity does not induce apoptosis in muscle fibers nor in inflammatory $\mathrm{T}$ cells: studies in patients with polymyositis, dermatomyositis, and inclusion body myositis. J Neuropathol Exp Neurol 55: 1205-1209

36 Dalakas MC (1998) Molecular immunology and genetics of inflammatory muscle diseases. Arch Neurol 55: 1509-1512

37 Bender A et al. (1998) T-cell heterogeneity in muscle lesions of inclusion body myositis. J Neuroimmunol 84: 86-91

38 Muntzing Ket al. (2003) Inclusion body myositis: clonal expansions of muscle-infiltrating $\mathrm{T}$ cells persist over time. Scand J Immunol 58: 195-200

39 Amemiya Ket al. (2000) Clonal restriction of T-cell receptor expression by infiltrating lymphocytes in inclusion body myositis persists over time:studies in repeated muscle biopsies. Brain 123: 2030-2039

40 Murata K and Dalakas MC (1999) Expression of the costimulatory molecule BB-1, the ligands CTLA-4 and CD28 and their mRNA in inflammatory myopathies. Am J Pathol 155: 453-460

41 Wiendel Het al. (2003) Muscle fibers and cultured muscle or cells express the $\mathrm{B} 7.1 / 2$ related costimulatory molecule ICOSL: implications for the pathogenesis of inflammatory myopathies. Brain 126: 1026-1035

42 Figarella-Branger D et al. (2003) Cytokines, chemokines, and cell adhesion molecules in inflammatory myopathies. Muscle Nerve 28: 659-682

43 De Bleecker JL et al. (2002) Differential expression of chemokines in inflammatory myopathies. Neurology 58: 1779-1785

44 Raju R et al. (2003) Expression of interferon-gamma inducible chemokines in the muscles of patients with inclusion body myositis. J Neuroimmunol 141: 125-131

45 Choi YC and Dalakas MC (2000) Expression of matrix metalloproteinases in the muscle of patients with inflammatory myopathies. Neurology 54: 65-71

46 Cupler EJ et al. (1996) Inclusion body myositis in HIV-I and HTLV-I infected patients. Brain 119: 1887-1893

47 Ozden S et al. (2004) Direct evidence for a chronic CD8 ${ }^{+}-$T-cell-mediated immune reaction to tax within the muscle of a human T-cell leukemia/lymphoma virus type 1-infected patient with sporadic inclusion body myositis. J Virol 78: 10320-10327

48 Saito M et al. (2002) Molecular analysis of T cell clonotypes in muscle-infiltrating lymphocytes from patients with human T lymphotropic virus type 1 polymyositis. J Infect Dis 186: 1231-1241 
49 Ferrer I et al. (2005) Involvement of clusterin and the aggresome in abnormal protein deposits in myofibrillar myopathies and inclusion body myositis. Brain Pathol 15: 101-108

50 Nagaraju Ket al. (2005) Activation of the endoplasmic reticulum stress response in autoimmune myositis: potential role in muscle fiber damage and dysfunction. Arthritis Rheum 52: 1824-1835

51 Grandea AG 3rd and Van Kaer L (2001) Tapasin: an ER chaperone that controls MHC class I assembly with peptide. Trends Immunol 22: 194-199

52 Lehner PJ and Cresswell P (2004) Recent developments in MHC-class-I-mediated antigen presentation. Curr Opin Immunol 16: 82-89

53 Ferrer l et al. (2004) Proteasomal expression, induction of immunoproteasome subunits, and local $\mathrm{MHC}$ class I presentation in myofibrillar myopathy and inclusion body myositis. J Neuropathol Exp Neuro/ 63: 484-498

54 Vattemi G et al. (2004) Endoplasmic reticulum stress and unfolded protein response in inclusion body myositis muscle. Am J Pathol 164: 1-7

55 Banwell BL and Engel AG (2000) aB-crystallin immunolocalization yields new insights into inclusion body myositis. Neurology 54: 1033-1041
56 Schmidt J et al. (2005) Distinct interplay between inflammatory and degeneration-associated molecules in sporadic IBM. Neurology 64 (Suppl 1): A331-A338

57 Wileman T (2006) Aggresomes and autophagy generate sites for virus replication. Science 312: $875-878$

58 Fratta $\mathrm{P}$ et al. (2005) Proteasome inhibition and aggresome formation in sporadic inclusion-body myositis and in amyloid-beta precursor proteinoverexpressing cultured human muscle fibers. Am J Pathol 167: 517-526

59 Greenberg SA (2001) DNA microarray gene expression analysis technology and its application to neurological disorders. Neurology 57: 755-761

60 Raju R and Dalakas MC (2005) Gene expression profile in the muscles of patients with inflammatory myopathies: effect of therapy with IVIg and biologic validation of clinical relevant genes. Brain 128: 1887-1896

61 Dalakas MC (2003) Therapeutic approaches in patients with inflammatory myopathies. Semin Neurol 23: 199-206

62 Lindberg C et al. (2003) Anti-T-lymphocyte globulin treatment in inclusion body myositis: a randomized pilot study. Neurology 61: 260-262

63 Dalakas MC (2006) Mechanisms of Disease: signaling pathways and immunobiology of inflammatory myopathies. Nat Clin Pract Rheumatol 2: 219-227

\section{Acknowledgments}

This research was supported by the Intramura Research Program of the $\mathrm{NIH}, \mathrm{NINDS}$

\section{Competing interests}

The author declared he has no competing interests. 